\title{
Pathology of the bone-cement interface in loosening of total hip replacement
}

\author{
U. E. Pazzaglia \\ Clinica Ortopedica dell'Universtà di Pavia, IRCCS Policlinico San Matteo, Via Taramelli, 3, I-27100 Pavia, Italy
}

Summary. The histopathology of the bone-cement interface in nine retrieved hip prostheses is reported. Three cases presented features similar to those observed by Charnley in highly successful implants, and they were classified as stable prostheses, although signs of periprosthetic bone resorption were already present. In this group a macrophagic reaction was evident even in the presence of a stable bone-cement interface, supporting the view that the release of particles by the cement or by the prosthetic components can precede the mechanical instability and be the primary cause of loosening. In six prostheses the connective tissue layer between the cement and the bone was thick and no bone trabecula reached the cement surface. The polymorphous features of this connective membrane probably resulted from instability and movement at the bone-cement and stem-cement interfaces. On the basis of the pathological changes observed in the study, the process of loosening must be far advanced before it is detected on radiographs; it is suggested that scintigraphy is the best noninvasive technique to demonstrate macrophage activation and increased bone remodeling around the cement in the early phases of loosening.

The etiology of prosthesis loosening is controversial: mechanical factors $[9,17]$, heat injury of the bone [12], inadequate fixation and cementing techniques $[1,8]$, allergy to implanted materials [5], and biological, reactive processes to particulate materials $[16,20,21]$ have been suggested. Most of these studies rest on the morphology of the bone-cement interface when the loosening process is far advanced and many factors have interacted to produce the pathological changes around the prosthesis. The scarce knowledge regarding the histology of early loosening and the sequence of events that follows explains the difficulty encountered in providing a definition of loosening and a meaningful interpretation of the clinical signs and radiographs.

In this study some of the aspects which were observed at the bone-cement interface suggest an early phase of loosening and offer an insight into the causes and the pathogenetic mechanism which lead to failure of the prosthesis.

\section{Materials and methods}

Nine patients underwent revision of their hip arthroplasty for pain or radiographic signs of loosening. Infection was excluded by routine laboratory tests, cultures of periprosthetic tissues for aerobic and anaerobic bacteria performed at the time of revision, and microscopic examination of histological sections. Scintigraphy was positive in all cases.

Data on patients are reported in Table 1 . In six cases CMW radiopaque cement had been used; in three there were no records of cement brands, but also in these the cement used was radiopaque. The prostheses in cases $1-3$, despite pain and progressive radiolucency around the cement in one, were not found to be grossly loosened at revision and osteotomy was required to remove the cement. In cases 4 and 5 a wide resection was performed and the entire proximal femur was available for the study. The femur and the bulk of cement were cut longitudinally with an oscillating saw in order to facilitate the removal of the stem. Transverse sections, $1 \mathrm{~cm}$ thick, were then cut with a band saw and fixed in neutral formalin $(10 \%)$. In the other cases only specimens which included cement, interface membrane, and a layer of bone were studied. Nineteen specimens, all from the femur, were technically acceptable. According to the site of biopsy, three classes were distinguished: one third proximal, one third middle and one third distal; they were further classified into medial and lateral biopsies.

Undecalcified specimens were embedded in methylmethacrylate resin and cut with a motorized Reichert microtome; sections were stained with hematoxylin-eosin and solochrome. Other specimens were decalcified in EDTA, gently freed from the cernent, and embedded in paraffin. Sections were stained with hematoxylin-eosin. An analytical study of particles contained in the cells was performed on routine histology sections; the coverslip was removed with xylene and the sections were then coated with a thin layer of gold or carbon and observed in a scanning electron microscope equipped for energy-dispersive X-ray analysis.

\section{Observations}

No significant differences were observed in specimens from different sites of the same case. On the contrary, 
Table 1. Clinical data of the nine removed prostheses

\begin{tabular}{|c|c|c|c|c|c|c|c|c|c|}
\hline Case & Group & Sex & $\begin{array}{l}\text { Age } \\
\text { (at } \\
\text { revision) }\end{array}$ & Diagnosis & $\begin{array}{l}\text { Pros- } \\
\text { thesis }\end{array}$ & $\begin{array}{l}\text { Pain-free } \\
\text { interval }\end{array}$ & Prosthesis & Cause of revision & $\begin{array}{l}\text { Histological } \\
\text { characterization } \\
\text { of macrophages }\end{array}$ \\
\hline 2. VB & A & M & 51 & Arthr. R. & $\mathrm{CH}$ & 12 years & 13 years & Pain, radiolucency & Metal particles \\
\hline 3. OM & A & $\mathrm{F}$ & 80 & Fract. L. & MU & 2 years & 5 years, 6 mos. & Pain & Polyethylene \\
\hline 6. NB & $\mathrm{B}$ & M & 61 & Arthr. R. & $\mathrm{CH}$ & 6 years & 8 years & Loosening & $\begin{array}{l}\text { Clear, granular } \\
\text { cytoplasm }\end{array}$ \\
\hline 7. PN & B & M & 74 & Arthr. R. & $\mathrm{LL}$ & 7 years, 1 mo. & 7 years, 1 mo. & Loosening & Polyethylene \\
\hline 8. CP & $\mathrm{B}$ & $F$ & 74 & Arthr. R. & $\mathrm{CH}$ & 1 year, 4 mos. & 1 year, 4 mos. & $\begin{array}{l}\text { Pain, local bone } \\
\text { resorption }\end{array}$ & $\begin{array}{l}\text { Clear, granular } \\
\text { cytoplasm }\end{array}$ \\
\hline
\end{tabular}

a Proximal femur resected and available for the study

CH, Charnley; LL, Lagrange-Letournel; MU, Müller; ST, Stanmore

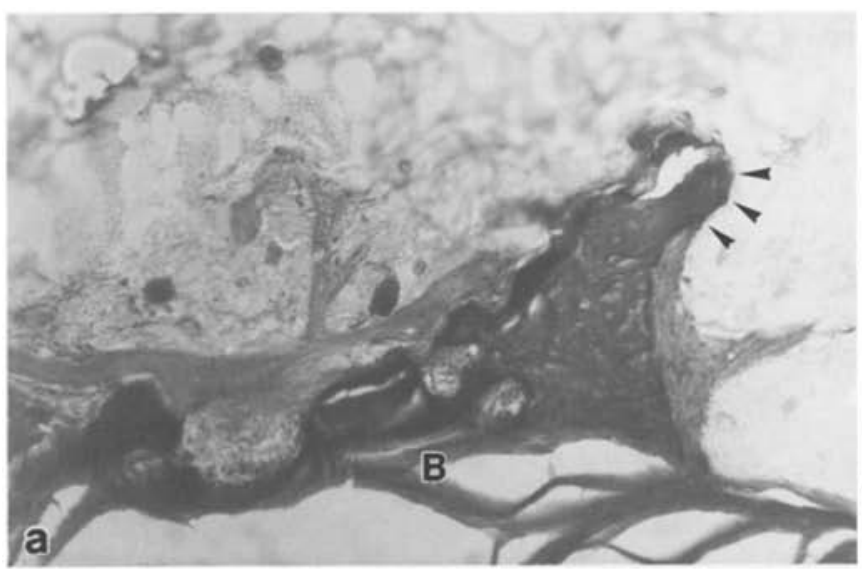

Fig. 1a, b. Case 1: section of undecalcified bone and cement; points of direct contact between acrylic cement and bone (arrows). $\mathbf{a}$ and $\mathbf{b}$ are details of Fig. 2 . Solochrome, $\times 100$

two groups of cases with common features were distinguished on the basis of histological findings, and they will be reported separately.

- Group A: at least two areas in each section of the bone-cement interface were present with aspects currently accepted as evidence for implant stability $[2,4]$.

- Group B: absence of any area of contact between cement and bone with an uninterrupted, thick connective layer.

In group A (cases 1-3) few points of direct contact between bone and cement were present (Fig. 1); most of the interface was formed by a connective tissue layer, which varied in thickness from $50 \mu \mathrm{m}$ to several millimeters (Fig. 2). Spindle-shaped fibroblasts and a dense col-

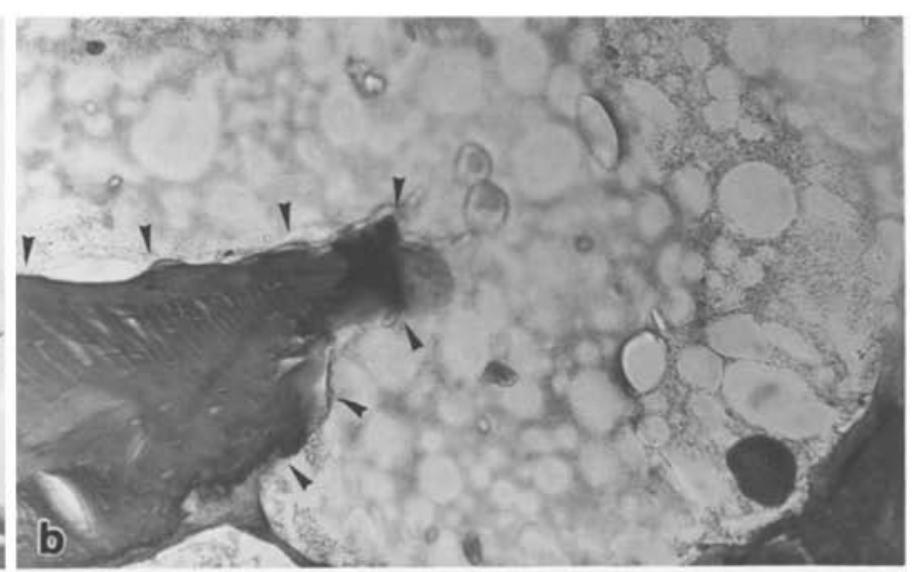

lagen fiber matrix were observed in the areas where the thickness was less (Fig. 2.1); as the latter increased the membrane became more cellular and marcrophages accumulated on the inner side in contact with the cement. Giant cells were also observed on the contour of the cement. Small fragments of lamellar bone were present within the soft-tissue membrane, indicating the original bony bed which had undergone remodeling and substitution by fibrous tissue (Fig. 2.2-4). In areas where the thickness reached $1 \mathrm{~mm}$ or more a definite pattern could no longer be recognized in the disposition of the dense connective and the cellular tissue, and sheets of macrophages penetrated into the haversian canals and medullary spaces of the bone; this was undergoing rapid remodeling with many resorption lacunae, which contained multinuclear osteoclasts or mononuclear cells of macrophagic type. Osteoblastic apposition was also enhanced.

In case 1 macrophages had a clear, granular cytoplasm, but no intracellular particles could be observed in 


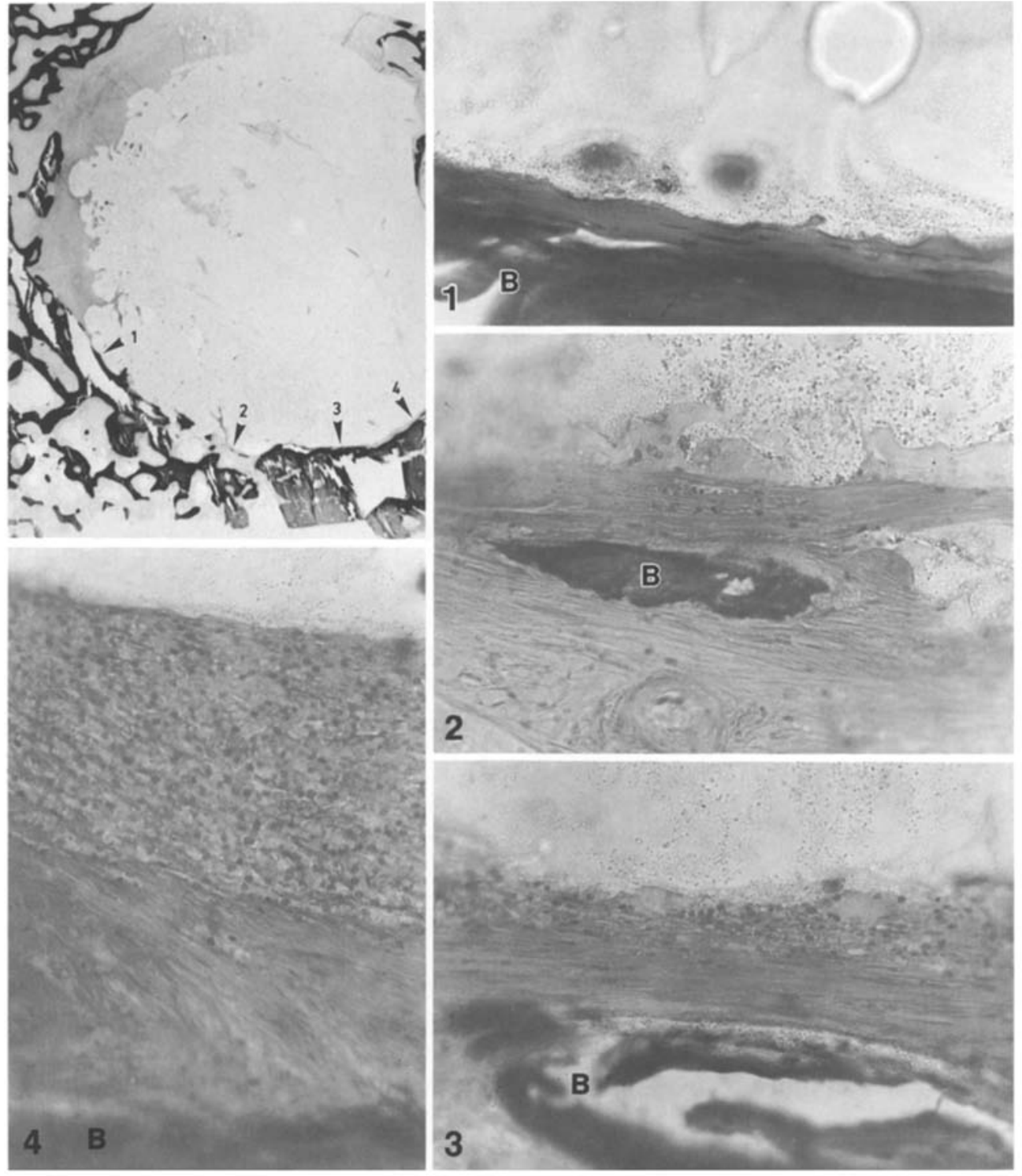

Fig. 2. Case 1: Section of undecalcified bone and cement. General view of the bone-cement interface in a specimen of the proximal femur; the connective membrane is thin on the lower and right side, while its thickness reaches several millimeters on the upper and left side. Points of direct contact are also present and are illustrated in Fig. 1. Solochrome, $\times 2.5$. Details of the interface at points indicated by arrows; $B$, bone; Solochrome, $\times 250$ : 1 Connective layer (about $50 \mu \mathrm{m}$ thick) is formed by flattened fibrocytes and bundles of collagen fibers. 2 Membrane is thicker and more cellular. Multi-nucleated giant cells are evident, in contact with the cement. A fragment of lamellar bone $(B)$ indicates the original bony bed, which had undergone resorption and substitution by fibrous tissue. 3 Inner layer of the membrane is formed by multi-nucleated giant cells and macrophages, outer by fibrous, dense connective tissue. 4 Thickness of membrane is further increased; the inner and outer layers are still well separated either bright field or polarized light. Macrophages contained opaque particles in case 2, and X-ray dispersive energy analysis gave the characteristic spectrogram for stainless steel (Fig. 3). In case 3 birefringent polyethylene particles were observed in macrophages and giant cells. In the latter two patients the cement surface was lined by flattened fibrocytes, and macrophages filled the medullary cavities of the bone interspersed within fat lobules. Zones of direct bone-cement contact and multinucleated giant cells were also present in these specimens (Fig. 4).

Distribution of macrophages in the sections showed two different patterns according to the type of cytoplasmic inclusions: clear, granular macrophages were denser in the neighborhood of the cement and gave the impression of a tissue front moving outward from the cement to the bone (Fig. 2); on the other hand, macrophages loaded with polyethylene or metal particles reached the bone-cement interface from the outside through vessels and capillaries (Figs. 5,6) and filled the medullary spaces and lacunae of the bone, but always a thin connective membrane or giant cells free from particles separated the cement from macrophagic cells.

In group B (cases 4-9) the connective tissue layer between the cement and the bone was uniformly thick and no bone trabecula reached the cement surface. Histologically, several types of tissue were observed, and they will be briefly summarized as follows:

1. Dense, fibrous tissue with flattened fibrocytes and bundles of densely crowded collagen fibers;

2. Loose, connective tissue with many vessels and sinusoids; the cells were of the fibroblastic type, but foci of macrophages and hemorrhagic areas were also present;

3. Amorphous, fibrin-like material;

4. Sheets of macrophages: granular as well as polyethylene or metal-filled cells were observed; although different inclusions may be present in the macrophages of 


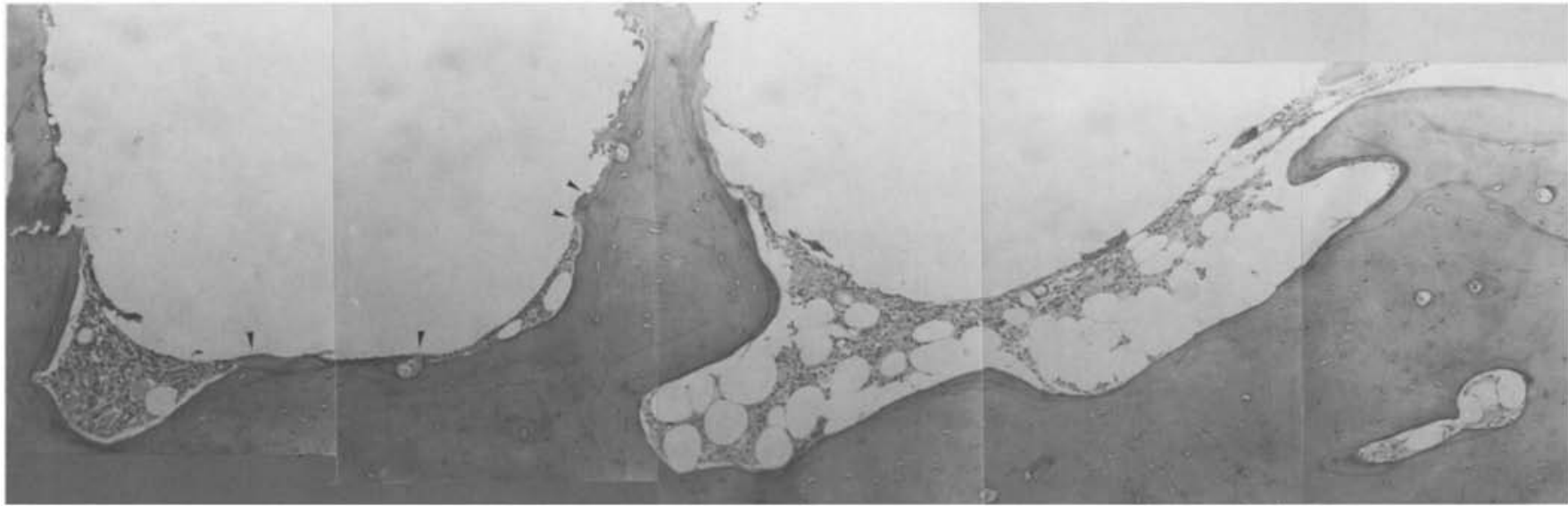

Fig. 3. Case 2: Decalcified section. Bone-cement interface in a specimen of the middle third of the diaphysis, medial side. Isolated points of direct bone-cement contact between arrows. A thin connective layer is present in the non-load-bearing zones, with multinuclear giant cells in contact with the cement. Marrow and vascular canals of the bone are invaded by mononuclear histiocytes. Hematoxylin-eosin, $\times 100$

each specimen, a prevailing type was recognizable in each case.

Scattered in these tissues were also necrotic bone debris, foci of calcification, acrylic pearls, and large polyethylene fibers. The different types of tissue were distributed in no particular order in the sections; however, amorphous fibrin-like material prevailed near the cement and the sheets of macrophages prevailed on the bone surface.

Remodeling of the bone was very active and the trabecula had a "moth-eaten" aspect. Macrophages filled old resorption lacunae and vascular spaces. These aspects coexisted with active osteoblastic apposition.

\section{Discussion}

In studies of the bone-cement interface from highly successful cases of total hip replacement, Charnley [2-4] showed that load transmission occurs at isolated points where there is direct contact between cement and bone or fibrocartilage. In the non-load-bearing zones a thin layer of connective tissue was observed: this delicate membrane also isolated the cement from the hemopoietic marrow and fatty tissue of the bone. Occasionally, histiocytes and giant cells were observed on the cement surface, but they were isolated from the marrow by the thin, connective membrane. It was postulated that these histological findings indicate a sound mechanical fixation of the prosthesis. No further data have been reported in the literature which are able to invalidate this assumption.

All the cases of this study involved unsuccessful prostheses with a clinical or radiographic diagnosis of loosening which required revision. Assignment of cases to group A or B was performed exclusively on the basis of histological findings.

The observation of areas which satisfy the histological criteria for stability in a biopsy specimen or even in a full-diameter section of the femur does not imply stability of the whole implant if breakage of the cement mantle has occurred; however, it can be assumed as evidence for stability in the segment examined.

The question of the relationship between mechanical factors and tissue reaction at the bone-cement interface is of paramount importance in understanding the phenomenon of loosening: macrophage reaction is a com-

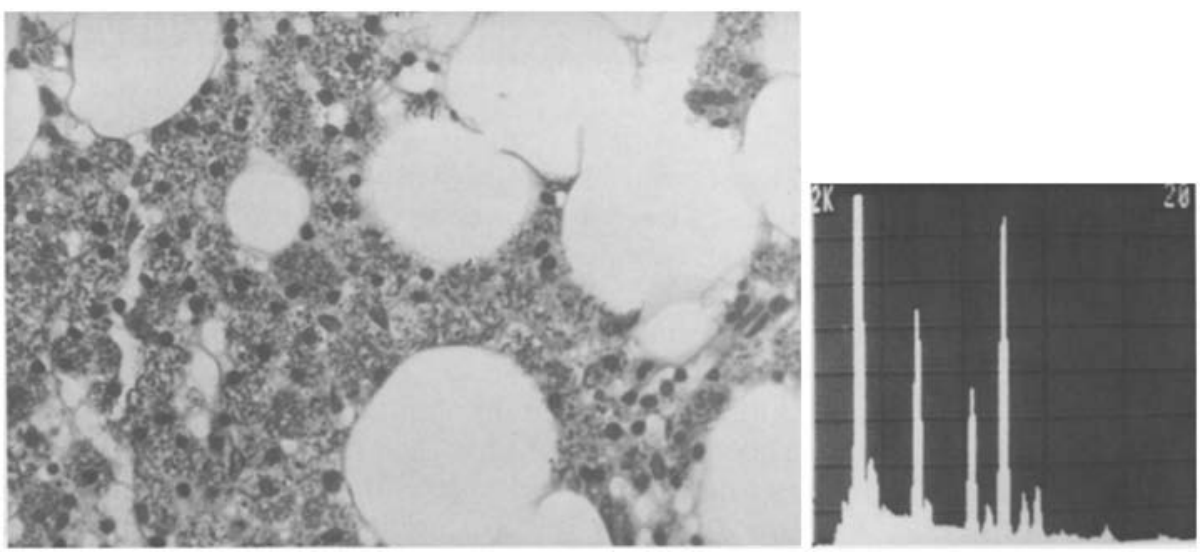

Fig. 4. Case 2: left Detail of Fig. 3. Macrophages loaded with opaque particles are observed between fat lobules of marrow. Hematoxylin-eosin, $\times 400$. right $\mathrm{X}$-ray dispersive-energy analysis gives the spectrogram of stainless steel 


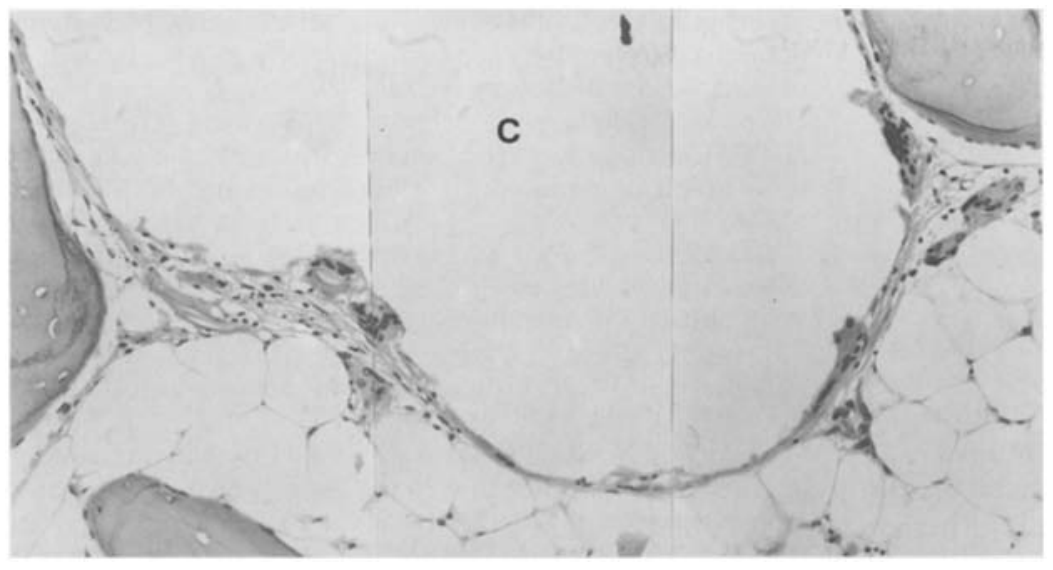

Fig. 5. Case 3: Decalcified section; bone-cement interface in a specimen from the proximal femur, lateral side. A thin connective layer separates the cement $(C)$ from the marrow. Two classes of multinucleated giant cells are observed, the first in contact with the cement, the other in the marrow. Hematoxylin-eosin, $\times 400$
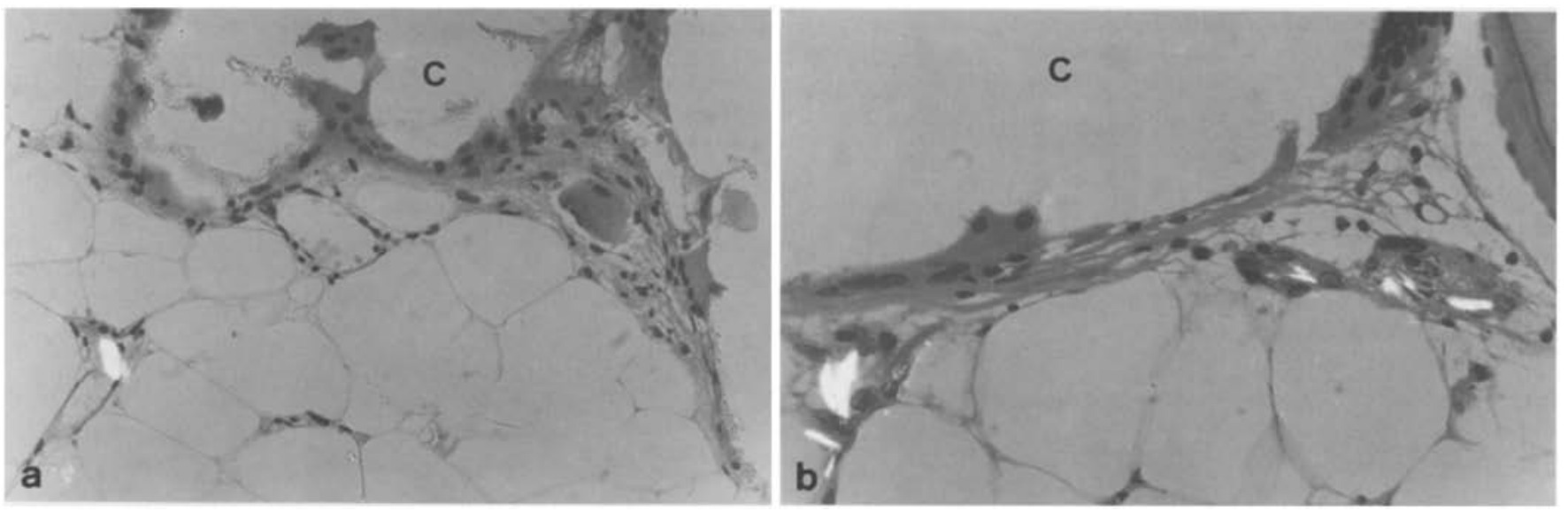

Fig. 6a, b. Case 3: Decalcified section; Details of Fig. 5. Multinucleated giant cells in contact with the cement $(C)$ do not contain birefringent particles. Giant cells in the marrow have phagocytosed polyethylene; in a the phagocytosing cell is observed inside the vessel lumen. Hematoxylin-eosin, double exposure in bright field and polarized light, $\times 400$

mon finding in all studies of loosened cemented implants $[13,16,18,21]$, but little evidence for their primary role in loosening has so far been produced [6]. Mechanical instability has been suggested as the factor triggering bone resorption and soft-tissue expansion around the cement: according to this hypothesis, macrophage reaction would be a phenomenon secondary to wear products of loosening or to necrotic, organic material formed by the sliding movements at the bone-cement interface [9]. The histological findings in the first group of this study suggest, on the contrary, that the macrophage reaction preceded the mechanical instability and was the primary cause of loosening in these cases.

Macrophage proliferation represents the tissue response to a variety of stimuli: among them metal and polyethylene particles are easily identified by analytical methods or by examination with polarized light [14]; no direct evidence of methylmethacrylate particles can be obtained, however, since they are solubilized during the processing of the specimens, and X-ray energy-dispersive analysis for barium (if radiopaque cement has been used) is not useful either since contamination of the tissue surrounding the cement may have occured in the process of cutting the sections. Also, in absence of direct evidence of methylmethacrylate inside the cytoplasm, the histological aspect of macrophages accumulating at the cement surface suggests a cellular response to methylmethacrylate in particulate form [21].

Although macrophages are not able to resorb the bone directly, osteoclasts are activated by the intense macrophagic reaction and the bone undergoes progressive resorption and fibrous substitution [15].

Polyethylene wear particles and metal corrosion particles may also initiate the macrophagic reaction; examples of this phenomenon were observed in group A: in these cases bone was invaded through vascular and medullary spaces in the absence of any communication between the tissue around the articulating surfaces of the prosthesis and the stable bone-cement interface. Socket wear, stem corrosion, or cement fragmentation represent, therefore, the source of particles which stimulate a continued macrophagic activity that is responsible for the bone resorption. This may produce either instability and migration of the implant when the loss of bone stock involves the whole surface of the cement, or localized osteolysis if the process is confined to limited areas [7]. 
The histological findings in group B are consistent with most reports of the bone-cement interface in loosened prostheses; necrotic bone debris, hemorrhagic areas in the connective tissue, amorphous fibrin-like material, and acrylic pearls are produced by the sliding movements of the unstable implant against the bone. Wear of the stem against the cement may become a source of metallic debris $[13,19]$. These are consequences of the instability and give no evidence of the primary cause of loosening.

Early changes at the bone-cement interface cannot be demonstrated by the currently employed, noninvasive investigations; the diagnosis of loosening is based on the detection of bone resorption around the implant by standard roentgenography or arthrography; other signs include migration of the prosthetic components or instability, which can be demonstrated by more sophisticated methods as such roentgen stereophotogrammetric analysis $[10,11]$. However on the basis of pathological changes at the bone-cement interface observed in this study, the process leading to loosening is far advanced when instability occurs, or before periprosthetic bone resorption may be detected on radiograms. This emphasizes the value of scintigraphy as the only noninvasive technique able to demonstrate macrophage activation and increased bone remodeling around the cement in the early phases of loosening.

Acknowledgements. The author is grateful to Dr. P. D. Byers and Miss M. Brown (Morbid Anatomy Dept., RNOH London) for their help in processing and cutting undecalcified sections.

\section{References}

1. Amstutz HC, Markolf KL, Mc Neice GR, Gruen TA (1976) Loosening of total hip components: cause and prevention. In: Proc 4th Open Scientific Meeting of the Hip Society. Mosby, St Louis, pp 102-116

2. Charnley J (1970) The reaction of bone to self-curing acrylic cement. A long-term histological study in man. J Bone Joint Surg [Br] $52: 340-353$

3. Charnley $\mathbf{J}$ (1975) The histology of loosening between acrylic cement and bone. J Bone Joint Surg [Br] 57:245-258

4. Charnley J (1979) Low- friction arthroplasty of the hip. Theory and practice. Springer, Berlin Heidelberg New York, pp 25-40

5. Evans EM, Freeman MAR, Miller AJ, Vernon Roberts B (1974) Metal sensitivity as a cause of loosening of the prosthesis in total hip replacement. J Bone Joint Surg $[\mathrm{Br}] 56: 626-$ 642
6. Freeman MAR, Bradley GW, Revell PA (1982) Observations upon the interface between bone and polymethylmethacrylate cement. J Bone Joint Surg [Br] 64:489-493

7. Harris WH, Schiller AC, Scholler JM, Freiberg RA, Scott R (1976) Extensive localized bone resorption in the femur following total hip replacement. J Bone Joint Surg [Am] 37 :612617

8. Lee AJC, Ling RSM, Vangola JS (1978) Some clinical variables affecting the mechanical behaviour of bone cement. Arch Orthop Trauma Surg 92:1-18

9. Linder I, Lindberg L, Carlsson A (1983) Aseptic loosening of hip prestheses. A histological and enzyme-histochemical study. Clin Orthop 175:93-104

10. Mjoberg B, Hansson LI, Selvik G (1984) Instability, migration and laxity of total hip prostheses. A roentgen stereophotogrammetric study. Acta Orthop Scand 55:141-145

11. Mjoberg B, Brismar J, Hansson LI, Pettersson $\mathbf{H}$, Selvic G, Onnerfalt R (1985) Definition of endoprosthetic loosening. Comparison of arthrography, scintigraphy and roentgenstereophotogrammetry in prosthetic hips. Acta Orthop Scand 56: $469-473$

12. Mjoberg B (1986) Loosening of the cemented hip prosthesis. The importance of heat injury. Acta Orthop Scand 57 [Suppl 221]:5-40

13. Pazzaglia UE, Ceciliani L, Wilkinson MJ (1985) Involvement of metal particles in loosening of metal-plastic total hip prostheses. Arch Orthop Trauma Surg 104:164-172

14. Pazzaglia UE, Dell'Orbo C, Wilkinson MJ (1987) The foreignbody reaction in total hip arthroplasties. A correlated light-microscopy, SEM and TEM study. Arch Orthop Trauma Surg 106:209-219

15. Pazzaglia UE, Pringle JAS (1988) The role of macrophages and giant cells in loosening of total joint replacement. Arch Orthop Trauma Surg 107:20-26

16. Revell PA, Weightman B, Freeman MAR, Vernon-Roberts B (1978) The production and biology of polyethylene wear debris. Arch Orthop Trauma Surg 81:167-177

17. Simon JR, Paul IL, Rose RM, Radin EL (1975) "Stiction-friction" of total hip prostheses and its relationship to loosening. J Bone Joint Surg [Am] 57:226-231

18. Vernon-Roberts B, Freeman MAR (1977) The tissue response to total joint replacement prostheses. In: The scientific bases of joint replacement. Pitman, Bath, pp 86-129

19. Willert HG, Ludwig J, Semlitsch M (1974) Reaction of bone to methacrylate after hip arthroplasty. A long-term gross, lightmicroscopic and scanning elecron-microscopic study. J Bone Joint Surg [Am] 56:1368-1382

20. Willert HG, Semlitsch M (1976) Tissue reactions to plastic and metallic wear products of joint endoprostheses. In: Gschwend $\mathrm{N}$, Bebrunner HV (eds) Total hip prostheses. Huber, Bern, pp 205-239

21. Willert HG (1977) Reactions of the articular capsule to wear products of artificial joint prostheses. J Biomed Mat Res 11: $157-164$ 